\title{
Isolamento das Veias Pulmonares para Tratamento da Fibrilação Atrial Paroxística. Resultados Clínicos após um Único Procedimento
}

\author{
Mauricio Scanavacca, Raul Sartini, Fernando Tondato, André d'Ávila, Denise Hachul, \\ Francisco Darrieux, Sissy Lara, Eduardo Sosa
}

São Paulo, SP

Objetivo - Avaliar a evolução clínica dos pacientes com fibrilação atrial paroxística submetidos a um único procedimento de isolamento das veias pulmonares.

Métodos - Estudados 49 pacientes consecutivos (36 homens; idade média de $53 \pm 10$ anos) com episódios freqüientes e sintomáticos de fibrilação atrial paroxística de difícil controle clínico. Para mapeamento da junção do átrio esquerdo com as veias pulmonares, foi utilizado o cateter decapolar circular Lasso e para ablação 30 watts e $50^{\circ} \mathrm{C}$, um cateter com ponta deflectivel e eletrodo distal de $4 \mathrm{~mm}$.

Resultados - Em seguimento médio de $12 \pm 5$ meses, 25 (51\%) pacientes não apresentaram recorrência de fibrilação atrial e 24 (49\%) apresentaram pelo menos uma recorrência. Em 20 (83\%), a $1^{a}$ crise ocorreu antes do $1^{\circ}$ mês e em 4, após 2 a meses. Após a introdução de drogas antiarrítmicas, 15 (63\%) pacientes apresentaram melhora importante, 10 tornaram-se assintomáticos, 5 referiam crises raras, auto-limitadas e de curta duração e $9(37 \%)$ permaneceram com as manifestações clínicas inalteradas, apesar das drogas antiarrítmicas e foram encaminhados à nova intervenção. No final do seguimento, 35 (71\%) pacientes permaneciam em ritmo sinusal estável sem recorrência de fibrilação atrial, após um único procedimento, $50 \%$ dos quais sem drogas antiarrítmicas.

Conclusão - A maioria dos pacientes com fibrilação atrial paroxística sintomática, não controlados com medicação antiarrítmica, obtém controle clínico após um único procedimento de isolamento das veias pulmonares.

Palavras-chave: fibrilação atrial, ablação por cateter, radiofreqüência, veias pulmonares

Instituto do Coração do Hospital das Clínicas - FMUSP

Correspondência: Mauricio I. Scanavacca - InCor - Unidade de Arritmias Cardíacas

Av. Dr. Eneas C. Aguiar, 44 - São Paulo, SP - Cep 05403-000

E-mail: mauricio.scanavacca@incor.usp.br

Recebido para publicação em 16/1/03

Aceito em 21/7/03
A ablação com radiofrequiência de focos ectópicos com origem nas veias pulmonares foi, recentemente, introduzida na prática clínica para tratamento de pacientes com fibrilação atrial paroxística ${ }^{1,2}$. Embora efetiva, esta técnica pode ser aplicada somente em uma minoria de pacientes com focos ectópicos muito ativos, o que torna possível sua localização durante o mapeamento eletrofisiológico ${ }^{3-5}$. Em pacientes com fibrilação atrial com mecanismo focal, mas de apresentação esporádica utilizaram-se manobras farmacológicas e eletrofisiológicas para induzir a ocorrência desses focos ectópicos durante o mapeamento ${ }^{6}$. Esta técnica revelou-se pouco efetiva ${ }^{7}$, pois as recorrências eram freqüentes durante o seguimento clínico. Durante procedimentos subseqüentes, observou-se foco ectópico em outras veias, não identificado no mapeamento inicial ${ }^{6}$. Assim, o isolamento empírico das quatro veias pulmonares, sem indução e localização dos focos ectópicos deflagradores da fibrilação atrial, vem sendo sugerido para tratar esses pacientes ${ }^{8-10}$. Contudo, os resultados clínicos, e a necessidade de novas intervenções ainda são controversos ${ }^{11-14}$. O objetivo deste estudo foi avaliar a evolução clínica de pacientes submetidos ao isolamento empírico de três a quatro veias pulmonares, após a realização de um único procedimento.

\section{Métodos}

Foram avaliados prospectivamente, de maio/01 a agosto/02, 49 pacientes com fibrilação atrial paroxística de difícil controle clínico, submetidos ao isolamento elétrico e empírico das veias pulmonares. Foram excluídos do estudo pacientes que apresentavam extra-sístoles atriais freqüentes ou taquicardias atriais que deflagravam fibrilação atrial durante o mapeamento eletrofisiológico, nos quais o procedimento seria dirigido ao foco deflagrador específico.

Eram 36 homens e 13 mulheres, com idade média de $54 \pm 10$ (variando entre 32 e 76) anos. Tinham diagnóstico de fibrilação atrial há 2 anos e usado pelo menos três drogas antiarrítmicas, sem sucesso, antes da indicação do proce- 
dimento. A fibrilação atrial foi considerada paroxística quando os episódios revertiam-se, espontaneamente, em $48 \mathrm{~h}$. Foram incluídos pacientes submetidos a cardioversão elétrica ou química, quando realizadas dentro de um período menor que $48 \mathrm{~h}$ do início da crise. Dos 49 pacientes, cinco apresentavam diagnóstico de insuficiência coronariana crônica (2 com infarto do miocárdio prévio); um apresentava miocardiopatia dilatada discreta; um hipertrofia septal assimétrica e os demais não apresentavam cardiopatia estrutural aparente. Sete pacientes tinham diagnóstico de hipertensão arterial sistêmica sob controle clínico. O tamanho médio do átrio esquerdo era de $40 \mathrm{~mm} \pm 6 \mathrm{~mm}$ (entre 29 e 50 $\mathrm{mm}$ ) e a fração de ejeção média do ventrículo esquerdo de $73 \pm 6 \%$. Os pacientes foram submetidos a anticoagulação oral por quatro a seis semanas (RNI entre 2,5 e 3,5) ou realizaram ecocardiograma transesofágico dentro de $24 \mathrm{~h}$ antes do procedimento para afastar trombo atrial.

Os pacientes foram encaminhados ao laboratório de eletrofisiologia após pelo menos $8 \mathrm{~h}$ de jejum. Foram submetidos à sedação com propofol intravenoso por anestesista. Foi realizada entubação orotraqueal ou instalada máscara laríngea, quando necessária. A monitorização consistiu da medida automática da pressão arterial não invasiva, oximetria e medida do $\mathrm{CO} 2$ expirado.

Três cateteres multipolares foram introduzidos por punção de veia femoral direita: um cateter octapolar ou decapolar (DAIG ou J\&J) foi introduzido no seio coronariano; um cateter decapolar circular (Lasso, Biosense Webster) foi introduzido por uma bainha longa no átrio esquerdo através de forame oval ou por punção transeptal. Um cateter quadripolar para ablação 7 ou 8 French, com espaço inter-eletrodos de $2 \mathrm{~mm}$ e eletrodo distal com $4 \mathrm{~mm}$ de diâmetro (EPT ou J\&J) foi também introduzido no átrio esquerdo, por uma segunda punção transeptal, quando necessária. Após as punções transeptais, procedeu-se à infusão de 10.000 UI de heparina intravenosa. Após 15min, foi realizado o tempo de coagulação ativado, repetido pelo menos a cada hora. Doses suplementares de heparina foram adicionadas com objetivo de manter o tempo de coagulação ativado entre 250 e 350 s. A angiografia das veias pulmonares com contraste (ioxalato de meglumina $32,5 \mathrm{~g} / 100 \mathrm{ml}$ ) foi realizada antes de se iniciar o isolamento das veias pulmonares, exceto a da veia pulmonar inferior direita, que não foi estudada sistematicamente. As bainhas transeptais (J\&J, DAIG e USCI) utilizadas para introduzir os cateteres eletrodos no átrio esquerdo foram usadas para este fim. As imagens fluoroscópicas pulsadas a 7 quadros/segundo foram obtidas com o equipamento Fisher (Fisher electrophysiology $X$-ray imaging system) na projeção oblíqua anterior esquerda $\left(30^{\circ}-45^{\circ}\right)$ para as veias pulmonares esquerdas e na projeção oblíqua anterior direita $\left(30^{\circ}\right)$ para as veias direitas. Neste sistema a mensuração do tempo de exposição ao raio X é restrita ao tempo de emissão dos pulsos de raio $X^{15}$. Eletrogramas bipolares filtrados na faixa de 80 e $600 \mathrm{~Hz}$ foram obtidos e gravados em sistema digital (EMS System - Netherlands).

A técnica para isolamento elétrico das veias pulmona- res consistiu no mapeamento da junção veno-atrial esquerda com o cateter Lasso, com objetivo de localizar os potenciais venosos pulmonares em conexão com o átrio esquerdo e desconectá-los com aplicações de radiofreqüência, com potência máxima de $30 \mathrm{We} 50^{\circ} \mathrm{C}$ por $30 \mathrm{a} 60 \mathrm{~s}$. O cateter Lasso era introduzido na veia pulmonar após uma angiografia e retirado até ficar aproximadamente a $5 \mathrm{~mm}$ de seu óstio (figura 1). O critério de isolamento da veia foi o desaparecimento dos potenciais elétricos de seu interior durante o ritmo sinusal ou estimulação atrial contínua (bloqueio de entrada). Para distinguir os potenciais das veias esquerdas dos eletrogramas da aurícula esquerda, o isolamento dessas veias foi realizado sob estimulação elétrica atrial contínua do seio coronariano distal. As veias pulmonares direitas foram mapeadas durante ritmo sinusal, sem estimulação atrial. A aplicação de radiofreqüência foi dirigida, inicialmente, para o ponto mais sugestivo da localização da conexão veno-atrial e mantida por 30s. O bloqueio da condução pela conexão foi demonstrado pelo alongamento na condução por este setor e pela mudança na sequiência de ativação no óstio da veia, analisada pela ativação no cateter Lasso (figura 2). As aplicações se repetiram nos outros pontos de conexão até o isolamento completo da veia pulmonar. Após a seqüência de 10 a 12 pulsos, foi realizada a avaliação da permeabilidade da veia pulmonar por angiografia com contraste. As aplicações foram interrompidas quando houve percepção subjetiva da redução do calibre do óstio da veia. $\mathrm{O}$ isolamento da veia pulmonar era considerado completo, quando os potenciais venosos obtidos pelo cateter Lasso desapareciam total ou parcialmente, quando havia redução desses potenciais, entretanto, sem seu desaparecimento completo. Angiografia das veias pulmonares foi realizada individualmente ao término de cada isolamento.

Após o término do procedimento, era feita infusão de 10 a $40 \mu \mathrm{g}$ de isoproterenol, seguida de adenosina (12 a $18 \mathrm{mg}$ ), na tentativa de identificar focos ectópicos deflagradores residuais. Os pacientes foram mantidos em repouso absoluto por 4 h e, em geral, receberam alta no dia seguinte. A enoxiparina subcutânea $(1 \mathrm{mg} / \mathrm{kg} 2 \mathrm{x} / \mathrm{dia})$ foi iniciada

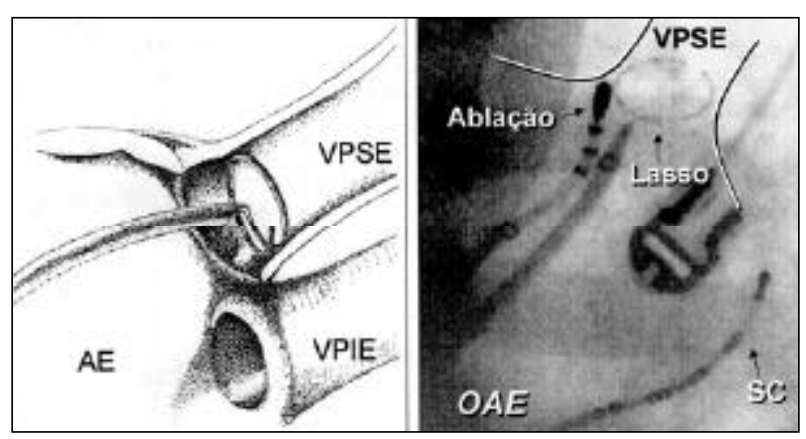

Figura 1 - Técnica de isolamento elétrico das veias pulmonares para tratamento da fibrilação atrial paroxística. A) representação esquemática da posição do cateter circular na veia pulmonar superior esquerda (VPSE); B) projeção fluoroscópica em oblíqua anterior esquerda (OAE) durante isolamento da VPSE. Note o cateter de ablação no óstio da VPSE. Os locais de ablação são selecionados a partir dos eletrogramas obtidos pelo cateter circular. AE- átrio esquerdo; VPIE - veia pulmonar inferior esquerda; SC - cateter posicionado no seio coronariano. 


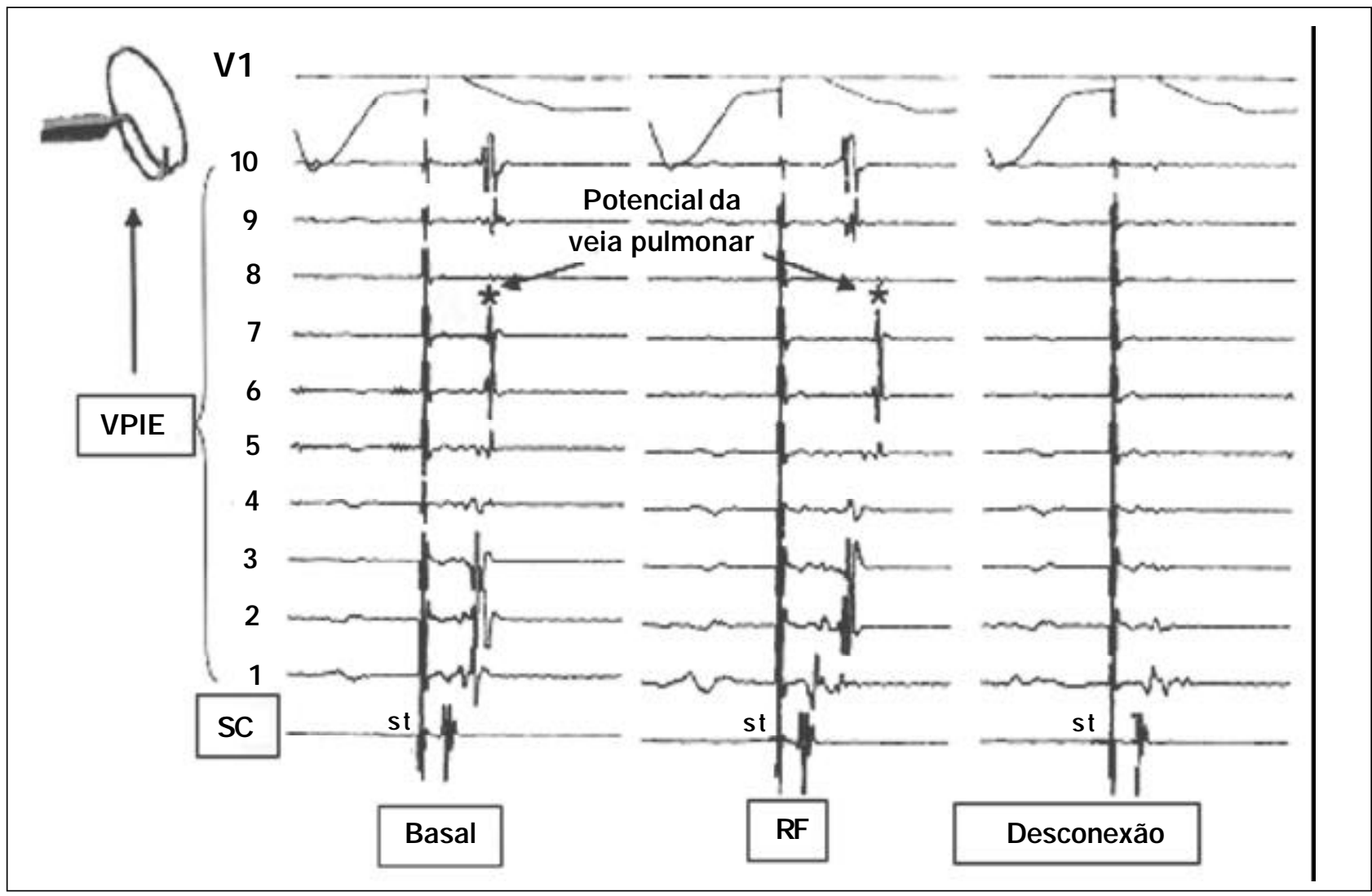

Figura 2 - Isolamento elétrico da veia pulmonar inferior esquerda (VPIE). Basal: eletrogramas bipolares de 1 (distal) a 10 (proximal) obtidos pelos eletrodos do cateter circular Lasso posicionado no óstio da VPIE durante estimulação elétrica (st) atrial realizada pelo par de eletrodos distais no seio coronário (SC). RF: note a mudança da seqüência de ativação do cateter circular durante aplicação de radiofreqüência (RF) secundária ao bloqueio parcial da entrada do estímulo elétrico no interior da VPIE. Desconexão: obtenção do isolamento elétrico da VPIE. Note o desaparecimento dos potenciais elétricos da VPIE no cateter Lasso. Os estímulos elétricos ativam o átrio esquerdo, confirmado pelo registro do cateter no SC, mas não conseguem penetrar no interior da VPIE.

após 6h do término do procedimento e mantida até anticoagulação oral atingir os valores de RNI preconizados $(2,5$ a 3,5). A manutenção do tratamento com antiarrítmico dependeu da recorrência dos sintomas dentro das primeiras $24 \mathrm{~h}$ ou da intensidade do desconforto provocado pelas crises de fibrilação atrial previamente à ablação.

O seguimento clínico foi realizado por consulta médica no ambulatório da Unidade Clínica de Arritmia do Instituto do Coração, por informação do médico que assistia ao paciente em seu consultório particular ou por contato telefônico com pacientes residentes fora da capital. O primeiro contato foi realizado um mês após o procedimento e repetido a cada três meses. Os pacientes foram interrogados a respeito da recorrência ou não dos sintomas. Em caso de recorrência, era sugerida a documentação eletrocardiográfica da crise. A monitorização ambulatorial (Holter e gravação de eventos sintomáticos) foi indicada quando o registro da crise no eletrocardiograma convencional não era possível. A repetição do procedimento foi indicada apenas aos pacientes que persistissem com recorrência de fibrilação atrial sintomática, apesar das drogas antiarrítmicas.

Para as comparações das variáveis contínuas adotouse o teste $t$ de Student e para a comparação das variáveis categóricas, o teste de Fisher. Em todos os testes, considerouse o nível de significância de $5 \%$.

\section{Resultados}

Foram abordadas 184 veias nos 49 pacientes (média de 3,7 veias por paciente). A duração média dos procedimentos foi de $231 \pm 47$ min e o tempo médio de exposição à radiografia pulsada a 7 quadros/s foi de $12 \pm 4 \mathrm{~min}$. Foram aplicados em média $21 \pm 12$ pulsos de radiofreqüência por veia: $25 \pm 14$ na veia pulmonar superior esquerda(VPSE); $22 \pm 10$ na superior direita (VPSD); $13 \pm 9$ na inferior esquerda (VPIE) e $23 \pm 12$ na inferior direita (VPID). Obteve-se isolamento completo da VPSEem 40 (82\%) pacientes, da VPSDem41 (84\%), da VPIEem 35 (81\%) e da VPID em 30 (67\%). Dos 49 pacientes, 18 obtiveram as quatro veias completamente isoladas, 20 três veias isoladas, 6 duas veias isoladas, 3 apenas uma veia foi isolada e, em 2, não foi obtido isolamento completo de nenhuma veia pulmonar.

Não houve complicações sérias durante o procedimento e no período de internação hospitalar. Nenhum paciente apresentou hematoma importante, complicações relacionadas à punção transeptal, manifestações clínicas de estenose de veias pulmonares ou evento embólico sistêmico ou pulmonar.

Em um período médio de $12 \pm 5$ meses de seguimento, $25(51 \%)$ pacientes não apresentaram recorrência de fibrila- 
ção atrial, sendo que $22(88 \%)$ tinham 3 ou 4 veias isoladas e $3(12 \%) 2$ ou menos veias isoladas. Dezenove pacientes permaneciam assintomáticos e seis referiam a sensação esporádica de extra-sístoles. Quinze pacientes $(60 \%)$ estavam sem medicações antiarrítmicas, 4 (16\%) ainda persistiam em uso de betabloqueadores, 3 devido a hipertensão arterial sistêmica e um devido à percepção de extra-sístoles e 6 (24\%) estavam em uso de amiodarona.

Vinte e quatro (49\%) pacientes apresentaram pelo menos uma recorrência de fibrilação atrial durante o seguimento. Em 20 (83\%), a primeira crise ocorreu antes de completar um mês e em 4 (17\%) após 2 a 9 meses de seguimento (figura 3). Após a introdução de drogas antiarrítmicas (betabloqueadores: 6, amiodarona: 6 , sotalol:3, propafenona: 2 , flecainide: um e bloqueador dos canais de cálcio: um), 15 (63\%) pacientes apresentaram melhora importante, 10 tornaram-se assintomáticos e 5 referiam crises raras, auto-limitadas e de curta duração. Quatro pacientes com recorrência precoce suspenderam as drogas antiarrítmicas após um a três meses da sua introdução e não voltaram a apresentar fibrilação atrial. Nove (37\%) pacientes permaneciam com as manifestações clínicas inalteradas, apesar das drogas antiarrítmicas e foram encaminhados à nova intervenção. No final do seguimento, 35 (71\%) pacientes permaneciam em ritmo sinusal estável, sem novas recorrências de fibrilação atrial, após um único procedimento, $50 \%$ dos quais sem drogas antiarrítmicas. Nenhum paciente apresentou manifestações clínicas de embolia sistêmica ou de estenose de veias pulmonares.

\section{Discussão}

A observação de que a fibrilação atrial paroxística é deflagrada por focos ectópicos com origem freqüente nas veias pulmonares abriu uma nova era na ablação por cateter, da fibrilação atrial. Contudo, a aplicação deste conceito ficou restrita a poucos pacientes, com focos ectópicos muito freqüentes e de fácil localização ${ }^{2-5}$. A tentativa de estender os potenciais benefícios clínicos da eliminação dos focos deflagradores para a ampla população de pacientes com fibrilação atrial paroxística apresentou dois inconvenientes: $1^{\circ}$ ) a dificuldade em localizar todos os focos, muitas vezes

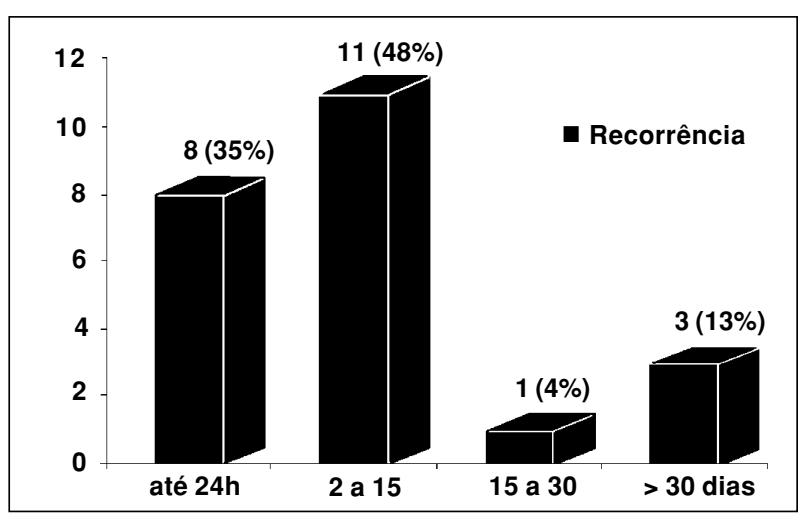

Figura 3 - Recorrência de fibrilação atrial após isolamento empírico das veias pulmonares. Observe que $19(82 \%)$ das 23 recorrências ocorreram dentro dos primeiros 15 dias após a ablação. ocultos e de difícil reprodução laboratorial, que resultaram em alta taxa de recorrência da fibrilaçao atrial e na realização de várias sessões de ablação na tentativa de controle das crises $^{6-8} ; 2^{\circ}$ ) o risco de provocar estenose das veias pulmonares ${ }^{16,17}$. Com objetivo de contornar essas limitações duas estratégias foram desenvolvidas a fim de isolar as quatro veias pulmonares em um único procedimento, com baixo risco de provocar estenose das veias pulmonares: o mapeamento e ablação de setores específicos do óstio ${ }^{8,9}$ e a reconstrução anatômica do átrio esquerdo com o sistema eletro-anatômico e ablação circunferencial dos óstios das veias pulmonares ${ }^{10,11}$.

A técnica de desconexão setorial desenvolvida por Haissaguerre e cols. e reproduzida neste estudo, visa isolar eletricamente as veias pulmonares minimizando o risco de uma estenose ${ }^{8,9}$. Baseia-se nas características anatômicas da junção veno-atrial, formada por conexões de tecido atrial que penetram nas veias em locais determinados ${ }^{18,19}$. Como cateter circular posicionado no óstio da veia, estas conexões podem ser identificadas e as aplicações de radiofreqüência mais restritas e de menor intensidade. Utilizando esta técnica, Haissaguerre e cols. relataram 3\% de estenose sintomática ${ }^{9}$. Oral e cols. utilizando a mesma técnica, não relataram complicações pulmonares ${ }^{12}$. Marrouche e cols. relataram estenose de veias pulmonares com aplicações no interior das veias ou com excessiva potência através de cateteres com ponta irrigada. Após o uso do ecocardiograma intracardíaco para controlar o local e a potência das aplicações, estenoses graves não foram mais observadas ${ }^{13}$. Em nossa casuística, nenhum paciente apresentou manifestações pulmonares relacionadas à estenose de veias. Vale salientar que a estenose das veias pulmonares manifesta-se apenas quando a redução do calibre do óstio é muito importante e, principalmente, quando há mais de uma veia acometida ${ }^{16,17}$. As manifestações clínicas ocorrem, em geral, nos primeiros dias após o procedimento, e já foi descrito relato de sua ocorrência após dois meses de evolução ${ }^{20}$. Não avaliamos sistematicamente a permeabilidade angiográfica das veias pulmonares durante o seguimento clínico, mas todos pacientes, exceto um, tiveram seguimento mínimo superior a seis meses e não apresentaram manifestações clínicas relacionadas a estenose de veias pulmonares. Estes dados iniciais indicam que a técnica de ablação setorial dos óstios das veias pulmonares com pulsos de radiofreqüência de baixa potência apresenta baixo risco de provocar uma estenose. Contudo, estudos a longo prazo são necessários para consolidar a segurança deste procedimento.

Os resultados clínicos do isolamento das veias pulmonares no tratamento de pacientes com fibrilação atrial ainda são controversos. A taxa de manutenção de ritmo sinusal estável sem recorrência de fibrilação atrial varia entre $70 \%$ a $94 \%{ }^{11-14,21}$. Os motivos para essas diferenças podem estar relacionadas com a seleção dos pacientes, a técnica de isolamento utilizada, o número de procedimentos realizados no mesmo paciente, uso de drogas antiarrítmicas e o modo de acompanhamento clínico dos pacientes ${ }^{22}$. Focos ectópicos externos aos óstios das veias pulmonares ou com origem em outras estruturas atriais têm sido relacionados com a re- 
corrência de fibrilação atrial após o isolamento das veias pulmonares ${ }^{23,24}$. No entanto, a reconexão de veias previamente isoladas parece ser a causa mais comum das recorrência e a obtenção de isolamento definitivo, sem o aumento do risco de estenose, é uma das questões técnicas a ser resolvida. Tem sido sugerido que a utilização de cateteres com ponta irrigada ou de $8 \mathrm{~mm}$ para produzir lesões mais profundas na borda atrial, evitando o interior das veias, relacionam-se com melhores resultados ${ }^{13}$. Entretanto, Macle e cols. utilizando cateteres com ponta irrigada realizaram 2 ou 3 procedimentos em $49 \%$ dos pacientes obtendo controle clínico em $80 \%$ deles, $44 \%$ com uso de antiarrítmicos ${ }^{14}$.

Merece destaque, o fato da maior parte das recorrências de fibrilação atrial ocorrer nas primeiras duas semanas do procedimento, sendo muitas delas transitórias e desaparecendo nas semanas seguintes. Esta observação também foi feita por outros autores ${ }^{13,25}$. Neste estudo, $50 \%$ dos pa- cientes com recorrência no primeiro mês após o procedimento não apresentaram novas crises de fibrilação atrial após 4 a 6 semanas, alguns mesmo após a suspensão das drogas antiarrítmicas. Este fenômeno poderia ser explicado por um processo inflamatório transitório e secundário à aplicação de radiofreqüência, assim como a evolução do processo de cicatrização da lesão ${ }^{26}$. Postergando uma nova intervenção por 4 a 6 semanas e restringindo-as aos pacientes sintomáticos e sem controle medicamentoso, indicamos um novo procedimento para apenas $16 \%$ dos nossos pacientes.

Concluindo, a maior parte dos pacientes com fibrilação atrial paroxística de difícil controle clínico obtém controle das crises após um único procedimento de isolamento das veias pulmonares. As recorrências, quando ocorridas, são mais freqüentes nas primeiras duas semanas, mas a maioria dos pacientes obtém controle clínico satisfatório durante o seguimento.

\section{Referências}

1. Haissaguerre M, Jais P, Shah DC, et al. Spontaneous initiation of atrial fibrillation by ectopic beats originating in the pulmonary veins. N Engl J Med 1998; 339: 659-66.

2. Chen SA, Hsieh MH, Tai CT, et al. Initiation of atrial fibrillation by ectopic beats originating from the pulmonary veins: electrophysiological characteristics, pharmacological responses, and effects of radiofrequency ablation. Circulation 1999; 100: 1879-86.

3. Scanavacca M, Sosa E, diAvila A, et al. Radiofrequency catheter ablation in patients with atrial fibrillation. Arq Bras Cardiol 1999; 72: 693-708.

4. Rocha Neto AC, Farias RL, de Paola AA. Treatment of atrial fibrillation with radiofrequency ablation and simultaneous multipolar mapping of the pulmonary veins. Arq Bras Cardiol 2001 Nov; 77: 407-28.

5. Mehta N, Távora MZ, Takeschita N, et al. Useful clinical features for the selection of ideal patients with atrial fibrillation for mapping and catheter ablation. Arq Bras Cardiol 2002; 78: 1-16.

6. Shah DC, Haissaguerre M, Jais P, et al. Curative catheter ablation of paroxysmal atrial fibrillation in 200 patients: strategy for presentations ranging from sustained atrial fibrillation to no arrhythmias. Pacing Clin Electrophysiol 2001; 24: $1541-58$

7. Gerstenfeld EP, Guerra P, Sparks PB, Hattori K, Lesh MD. Clinical outcome after radiofrequency catheter ablation of focal atrial fibrillation triggers. J Cardiovasc Electrophysiol 2001; 12: 900-8.

8. Haissaguerre M, Jais P, Shah DC, et al. Electrophysiological end point for catheter ablation of atrial fibrillation initiated from multiple pulmonary venous foci. Circulation 2000; 101: 1409-17.

9. Haissaguerre M, Shah DC, Jais P, et al. Electrophysiological breakthroughs from the left atrium to the pulmonary veins. Circulation 2000; 102: 2463-5.

10. Pappone C, Rosanio S, Oreto G, et al. Circumferential radiofrequency ablation of pulmonary vein ostia: A new anatomic approach for curing atrial fibrillation. Circulation 2000; 102: 2619-28.

11. Pappone C, Oreto G, Rosanio S, et al. Atrial electroanatomic remodeling after circumferential radiofrequency pulmonary vein ablation: efficacy of an anatomic approach in a large cohort of patients with atrial fibrillation. Circulation 2001; 104: 2539-44

12. Oral $\mathrm{H}$, Knight BP, Tada $\mathrm{H}$, et al. Pulmonary vein isolation for paroxysmal and persistent atrial fibrillation. Circulation 2002; 105: 1077-81.

13. Marrouche NF, Dresing T, Cole C, et al. Circular mapping and ablation of the pul- monary vein for treatment of atrial fibrillation: impact of different catheter technologies. J Am Coll Cardiol 2002; 40: 464-74.

14. Macle L, Jais $P$, Weerasooriya $R$, et al. Irrigated-tip catheter ablation of pulmonary veins for treatment of atrial fibrillation. J Cardiovasc Electrophysiol 2002; 13 1067-73.

15. Scanavacca M, d'Avila A, Velarde JL, Reolao JB, Sosa E. Reduction of radiation exposure time during catheter ablation with the use of pulsed fluoroscopy. Int $\mathrm{J}$ Cardiol 1998; 63: 71-4.

16. Robbins IM, Colvin EV, Doyle TP, et al. Pulmonary vein stenosis after catheter ablation of atrial fibrillation. Circulation 1998; 98: 1769-75.

17. Scanavacca M, Kajita LJ, Vieira M, Sosa EA. Pulmonary vein stenosis complicating catheter ablation of focal atrial fibrillation. J Cardiovasc Electrophysiol 2000; 11: 677-81

18. Cabrera JA, Sanchez-Quintana D, Farre J, et al. Implications for catheter ablation. Ultrasonic characterization of the pulmonary venous wall: echographic and histological correlation. Circulation 2000; 101: 1274-81.

19. Ho SY, Cabrera JA, Tran VH, Farre J, Anderson RH, Sanchez-Quintana D. Architecture of the pulmonary veins: relevance to radiofrequency ablation. Heart 2001; 86: 265-70.

20. Sohn RH, Schiller NB. Left upper pulmonary vein stenosis 2 months after radiofrequency catheter ablation of atrial fibrillation. Circulation 2000; 101: E154-5.

21. Shah DC, Haissaguerre M, Jais P, et al. Electrophysiologically guided ablation of the pulmonary veins for the curative treatment of atrial fibrillation. Ann Med 2000; 32: 408-16.

22. Turco JP. Atrial fibrillation ablation: Role of patient enrollment and follow-up in clinical results. J Am Coll Cardiol 2003; 41: 1232-3.

23. Tsai CF, Tai CT, Hsieh MH, et al. Initiation of atrial fibrillation by ectopic beats originating from the superior vena cava: electrophysiological characteristics and results of radiofrequency ablation. Circulation 2000; 102: 67-74.

24. Hwang C, Wu TJ, Doshi RN, Peter CT, Chen PS. Vein of Marshall cannulation for the analysis of electrical activity in patients with focal atrial fibrillation. Circulation 2000; 101: 1503-5.

25. Oral H, Knight BP, Ozaydin M, et al. Clinical significance of early recurrences of atrial fibrillation after pulmonary vein isolation. J Am Coll Cardiol 2002; 40 $100-4$

26. Fenelon G, Brugada P. Delayed effects of radiofrequency energy: mechanisms and clinical implications. Pacing Clin Electrophysiol 1996; 19: 484-9. 